\title{
Resonant Beam Steering and Carpet Cloaking Using an Acoustic Transformational Metascreen
}

\author{
M. Amin,,${ }^{1, *}$ O. Siddiqui, ${ }^{1}$ W. Orfali, ${ }^{1}$ M. Farhat, ${ }^{2}$ and A. Khelif ${ }^{3}$ \\ ${ }^{1}$ College of Engineering, Taibah University, P.O. Box 344, Madinah 41411, Saudi Arabia \\ ${ }^{2}$ Division of Computer, Electrical, and Mathematical Sciences and Engineering, King Abdullah University of \\ Science and Technology, Thuwal 23955-6900, Saudi Arabia \\ ${ }^{3}$ Institut FEMTO-ST, CNRS, Université de Bourgogne Franche-Comté, 15B Avenue des Montboucons, 25030 \\ Besanon, France
}

(Received 3 August 2018; revised manuscript received 11 November 2018; published 12 December 2018)

\begin{abstract}
We invoke the Huygens principle to derive dispersion characteristics of acoustic transformational metasurfaces that can deflect parallel wavefronts in a desired direction. We also propose a dual-Lorentz resonator whose aperture fields can be tuned by geometrical changes to implement a particular phase with unity reflection coefficient. The proposed metascreen is designed by our arranging slightly detuned Lorentz cavities that generate the necessary interference to compensate for the incident wavefronts. Since a complete $0-2 \pi$ range of the reflection phase is achieved, the metascreen can steer a beam across the full horizon. Moreover, since the proposed dual resonator has subwavelength dimensions, the metascreen is capable of high-resolution imaging, which is an essential trait in cloaking and deceptive acoustics. Successful demonstration of acoustic beam steering and carpet cloaking is done for a wavelength of $790 \mathrm{~mm}$ with the help of full-wave simulations.
\end{abstract}

DOI: 10.1103/PhysRevApplied.10.064030

\section{INTRODUCTION}

The discovery of photonic crystals kindled interest among the scientific community in engineered electromagnetic structures [1-3]. Since the unit-cell dimensions of these periodic structures (i.e., the periodicity) is on the order of a wavelength, most of the interesting wave effects, such as the photonic band gaps, were observed in the higher-order spectra [4]. The advent of metamaterials can be regarded as a milestone in the field of dispersion engineering that introduced the design of true artificial bulk media that possess properties beyond those of naturally occurring materials. The term "metamaterial" was initially coined to represent the periodic structures that demonstrate a negative refractive index in their fundamental Brillouin zone $[5,6]$. Later, the term was generalized to also encompass other artificial structures with unique electromagnetic properties. Examples include plasmonic [7], chiral [8], and photonic $[9,10]$ metamaterials. Similar phenomena were observed in photonic crystals in the near-to-wavelength optical regimes [11,12].

On the parallel lines, taking into account the equivalence of the acoustic wave dynamics and the Maxwell equations [13], analogous dispersive phenomena were also revealed in classical structures that support mechanical and acoustic

\footnotetext{
*masharif@taibahu.edu.sa
}

waves [14-22]. Quite extraordinarily, the possibility of overcoming the diffraction limit was explored in a slab of double-negative acoustic metamaterial that can image both the far-field and the near-field modes [22-27].

The two-dimensional metamaterial counterpart, known as a "metasurface" for its unusual dispersive properties, was the subject of intense research in previous decades [28-30]. The usefulness of a metasurface stems from its property of subwavelength periodicity and thickness that make it a low-profile interface that introduces a sudden change in amplitude and phase. Since metasurfaces are periodic structures, most of their applications are based on their property of spectral selection, such as filtering metasurfaces [30,31] and optical and acoustic absorbers $[32,33]$. The concept of a metasurface is more general and is applied to a wide range of applications. The earlierintroduced artificial impedance surfaces have similar dispersion characteristics but were primarily used to suppress the surface waves [34].

More recently, Huygens surfaces [35] were introduced as a class of nonperiodic metasurfaces in which the adjacent cells are slightly different from each other so that a particular phase profile can be synthesized. The idea is rooted in the Huygens principle and its field equivalence suggested by Schelkunoff [36]. The dispersion can be tailored by forcing a specific current or field phase distribution that allows the construction of an arbitrary 
shaped wavefront close to the boundary of the metasurface, leading to beam forming and focusing [35,37]. Not long ago, an acoustic Fresnel reflector [38] and a conformal metasurface for illusion acoustics [39] were suggested. These two recent publications present a similar method of wavefront synthesis but exploit two different types of detuned resonant unit cells. The unit cells used by Dubois et al. [39] were cylindrical Helmholtz-type resonators that have considerable absorption. On the other hand, Amin et al. used air-filled slit-shaped cavities constructed with rigid acoustic material that are matched to free space with no significant absorption, leading to near-unity reflection coefficients. Furthermore, since these unit cells are operated in the Fano-resonance band marked by sharp phase profiles [40-47], the adjacent cells have almost identical dimensions, leading to a low-profile flat metascreen geometry [38].

In this paper, we present a general method to reconstruct the reflected wavefront in a certain direction by first deriving a generalized dispersion equation of a Huygens acoustic metasurface. Subsequently, detuned binary resonant unit cells are used to construct the required dispersion profile for beam-steering and cloaking applications. The metasurface is phase modulated by our varying the height of the adjacent resonator, resulting in subwavelength unit-cell dimensions of approximately $\lambda / 5 \times \lambda / 12$. An analogous wavefront-synthesis method has been used traditionally by antenna engineers to design reflect arrays in which adjacent antenna elements (usually resonant patches) are phase shifted by implementation of slightly different geometries [48]. In the case of reflect arrays, however, the antenna elements are separated by the order of a wavelength and hence the beam forming is executed by the superposition and cancellation of electric fields in desired directions [49]. In the case of Huygens surfaces, the phase correction is applied over the subwavelength order and hence the resulting wavefronts have significantly higher resolution, which is an essential requirement in applications such as cloaking and other forms of illusion acoustics [39].

\section{DISPERSION EQUATION OF A REFLECTING HUYGENS METASURFACE}

Consider the generalized problem of wavefront reflection in Fig. 1(a), where a Huygens metasurface placed perpendicular to the incident acoustic rays causes the reflected wavefronts to propagate at an arbitrary angle $\theta$. Alternatively, as depicted in the phase space [Fig. 1(b)], each ray would gain an additional phase of $k(x) d^{\prime}$ inside the unit cell it impinges on. Invoking the Huygens principle, we can rearrange the secondary wave sources so that a planar wavefront is created at an inclined angle $\theta$. Therefore, the two acoustic paths shown in Fig. 1(b) should be equal:

$$
\frac{k_{\min } d^{\prime}}{\omega}+\frac{k_{o} D \sin \theta}{\omega}=\frac{k(x) d^{\prime}}{\omega}+\frac{k_{o}(D-x) \sin \theta}{\omega} .
$$

(a) Reflection ray diagram

(b) Calculation of dispersion equation

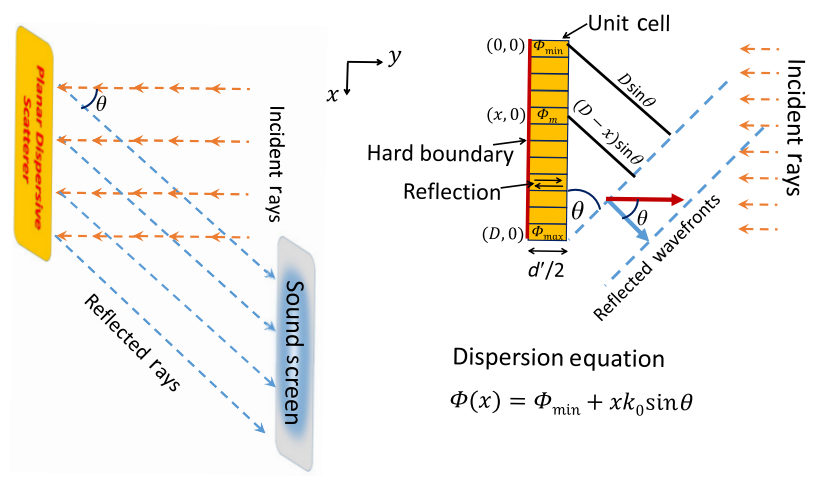

FIG. 1. Reflection from a flat Huygens surface. (a) Mechanism of reflection showing the phase $(k)$ vectors. (b) The phase space showing the incident and reflected wavefronts and basis for the dispersion equation. The resulting dispersion equation is indicated at the bottom.

Equating $k(x) d^{\prime}=\phi(x)$ and $k_{\min } d^{\prime}=\phi_{\min }$, we can obtain the following dispersion equation:

$$
\phi(x)=\phi_{\min }+x k_{o} \sin \theta .
$$

To demonstrate the wave propagation in the presence of the metasurface, we simulate a metasurface at a frequency of $434 \mathrm{~Hz}$ with a sufficiently large aperture of approximately $40 \lambda$ to create planar wavefronts. Isotropic point sources of unity amplitude are placed at the unit-cell locations with the modulated phase obtained from Eq. (2). A superposition of the Fourier components is calculated at $434 \mathrm{~Hz}$, and the phases of the vectorially combined acoustic pressure field are plotted in Fig. 2 for two cases of reflection angles. As shown, the reflected wavefronts travel in the directions required by the dispersion equation. The fact that the reflected wavefronts are deflected in the desired directions indicates a powerful dispersion-based method of wave manipulation that can be exploited in the art of deception and camouflage [39,50]. Hence an arbitrary virtual surface can be created with manipulated reflected wavefronts to delude detection radars or to cloak sensitive targets.

\section{THE ACOUSTIC METASCREEN: HUYGENS-WAVEFRONT SYNTHESIS}

It is a challenging task to design an acoustic reflector capable of inducing highly dispersive effects that would result in an arbitrary Huygens wavefront. Here we propose detuned Lorentz resonators that are based on the wave-interference principles to form the sharp phase characteristics that are required to generate the subwavelength dispersive effects. In a conventional homogeneous material, similar steep phase profiles occur in the spectral vicinity of absorption lines, as necessitated by 


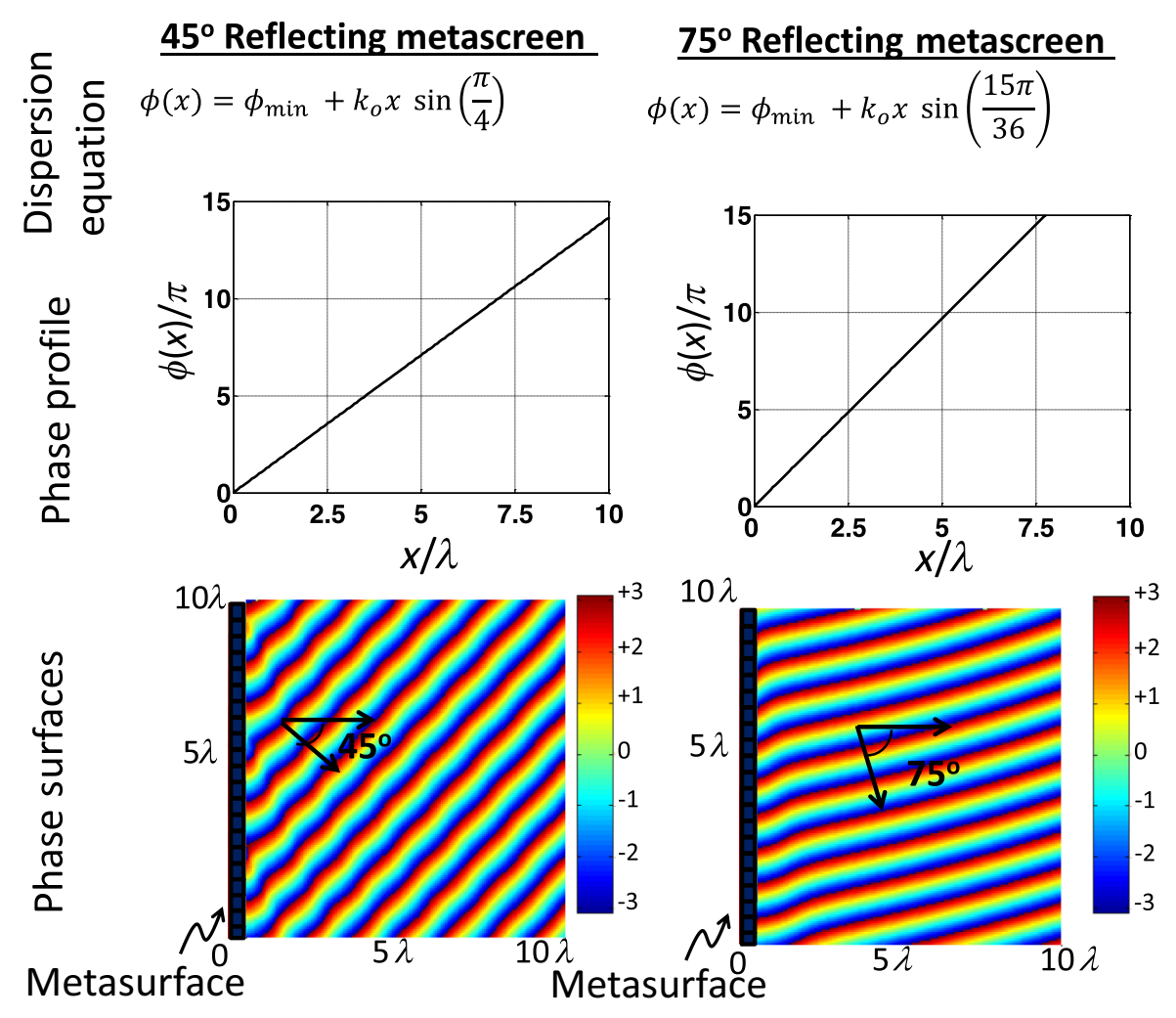

FIG. 2. The top row shows the dispersion equation for a $45^{\circ}$ and a $75^{\circ}$ metascreen. The center row shows the dispersion profile with the unit-cell phase as a function of the metascreen $x$ dimension. The bottom row shows a beam-steering application: the phase of the reflected wavefronts indicating the propagation of the reflected wave in directions at $45^{\circ}$ and $75^{\circ}$ (the incident phases are not shown).

the Kramers-Kronig relations [51]. On the other hand, the proposed cavity-based metascreens work in reflection mode, which is devoid of any absorption losses and hence leads to a power-efficient interference mechanism to achieve the dispersive effects. Moreover, since the acoustic resonators are mechanically tunable, the proposed method offers dynamic control over the shape of the reflected wavefronts that could lead to many exciting new applications.

Dispersion-based acoustic scattering requires efficient control over the reflected field phase while providing minimum nonradiative losses [38,39]. Furthermore, to obtain a decent transformational resolution, the unit cells should be of subwavelength dimensions. We show in this section that an interference phase profile created by two detuned acoustic cavities in close proximity can satisfy these two conditions of lossless scattering and subwavelength resolution and hence can be used in our metasurface design. To understand the interference phenomenon, let us first consider the phase profile formed by the waves reflected from an infinite array of identical Lorentz acoustic cavities (unit cells), as shown in Fig. 3(a). The reflection coefficient of the given cavity array is calculated by full-wave simulations with COMSOL MULTIPHYSICS (the simulation setup is provided in Supplemental Material [52]). Since the cavities are made from acoustically hard material forming a perfect reflector, it can be seen from Fig. 3(b) that the resulting reflectance is near unity over the entire spectrum. However, in the absence of an interference mechanism, the resulting phase spectrum [Fig. 3(c)] has a linear profile with a change of only $0.3 \mathrm{rad}$ in the desired frequency

(a)

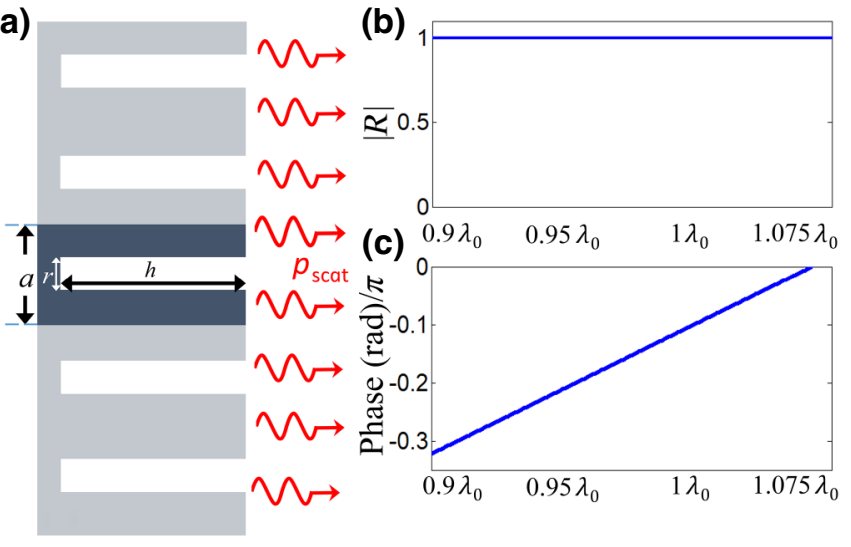

FIG. 3. (a) The Lorentz-cavity array on top of a rigid substrate. The height $(h)$ of the cavity is $8 / 3 a$ and the period $(a)$ of the periodic array is given by $7 / 79 \lambda_{0}$. (b) The reflectance amplitude and (c) its phase relative to the change in the wavelength $\lambda_{0}$. 
range. In quantum mechanical terms, the periodic array of identical resonators behaves as a dispersionless medium with the characteristic bright mode that couples directly with the incident pressure fields and can be regarded as the continuum state of the atomic structure.

Next we consider the interference from two slightly different (spectrally detuned) Lorentz cavities, which are collectively termed a "binary cell." From the quantum mechanical perspective, this second cavity embedded within the binary cell supports an analogous dark mode that must be spectrally detuned to be efficiently coupled to the earlier-observed bright mode. Consider Fig. 4(a), in which a periodic array of such binary cells is formed by our combining two slightly different Lorentz cavities . The detuning effect in a binary cell leading to the resonant wave interference can be obtained by our varying either the width $\left(r_{1} / r_{2}\right)$ or the height $\left(s_{i}=h_{i} / h\right)$ of the adjacent resonator. Henceforth, when an acoustic wavefront is incident on the binary-cell array, the resulting reflection phase profile [Fig. 4(b)] contains a significantly steeper phase variation compared with the single-cavity array (indicated by a dotted line). As expected, the corresponding resonances shift to lower wavelengths (blueshift) when the relative cavity widths $r_{1} / r_{2}$ are reduced. It can be further observed in Fig. 4(b) that the ratio of $r_{1} / r_{2}=2 / 7$ in this particular design gives the maximum phase-variation range around the design wavelength (here assumed to be $790 \mathrm{~mm}$ ), and hence it represents the most-suitable value for the metasurface design. From the design perspective, it is important to keep all the constituting binary cells of constant width so that they are uniformly illuminated by the incident wavefront. Therefore, to construct the spatial-dispersion relation [Eq. (2)] at a particular design wavelength $\lambda_{0}$, we scale the cavity heights (the parameter $s_{i}$ ) for the periodic binary-cell array in Fig. 4(a). The reflected phase incurred at the design wavelength is plotted against the scaled height in Fig. 4(c). A full $2 \pi$ phase spectrum is obtained, corresponding to scaled heights ranging between 0.95 and 1.2. Video 1 provides the frequencydependent relationship between the reflection coefficient of the periodic binary-cell array and the corresponding sound-pressure-field distribution inside the unit cell.

Since every binary cell on the metascreen can be mapped to the phase profile of Fig. 4(c), the smoothly varying graph corresponds to the enhanced resolution of the resulting wavefront. The relatively smaller range of the scaled heights that provide a full phase swing indicates that the binary cells do not significantly differ from each other, leading to compact and flat metascreen designs. This fine resolution that is needed to construct coherent wavefronts with no phase discontinuities between adjacent cells results from the subwavelength period $a$ (lattice constant) (a)

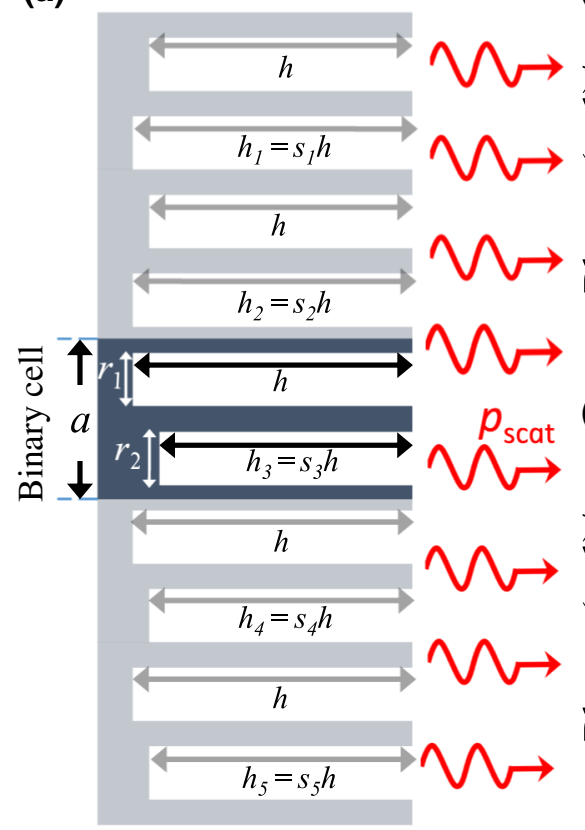

(b)

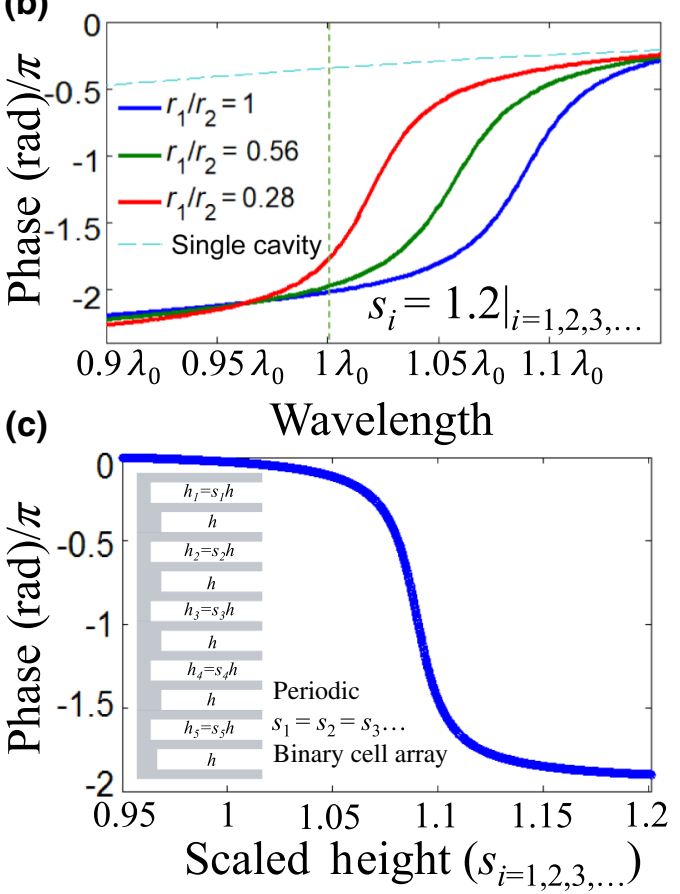

FIG. 4. (a) The periodic binary-cell array on top of a rigid substrate. The height $h=8 / 3 a$ is kept constant while the height $\left(h_{i}\right)$ of the alternate cell is scaled with respect to the fixed cavity. The period of the binary unit cell is $a=7 / 79 \lambda_{0}$. The side-wall thickness is $t=1 / 5 a$, and the ratio of the width of cell cavities is $r_{1} / r_{2}=2 / 7$. (b) Reflection phase relative to variation of the wavelength. (c) Reflection phase for a fixed wavelength of $\lambda_{0}=790 \mathrm{~mm}$ and scale-height $\left(s_{i}\right)$ variation between 0.95 and 1.2 . The inset shows the periodic binary-cell array used in (b),(c). 


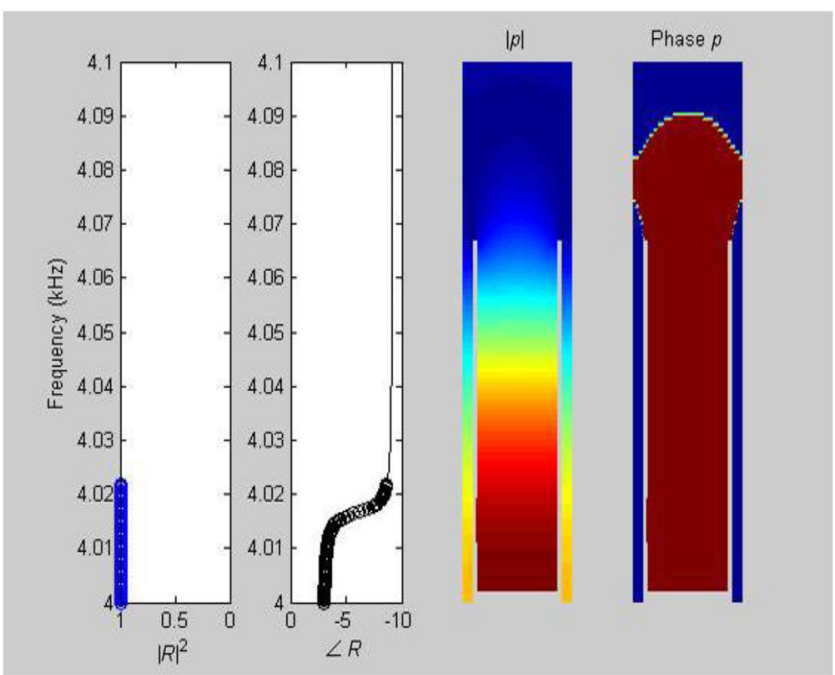

VIDEO 1. Dispersion visualized inside the unit cell of the periodic binary-cell array by showing the frequency-dependent relationship between the phase of reflection coefficient and corresponding sound-pressure-field distribution.

of the binary-cell Lorentz array. For example, in the current design, the binary cells are dimensioned in such a way that the ratio of the wavelength to the lattice constant $(\lambda / a)$ remains above 11 .

\section{CLASSICAL COUPLED-RESONATOR MODEL OF THE DISPERSIVE BINARY CELL}

Since the proposed concept of the acoustic metascreen is based on the principle of interference, its basic constituent (i.e., the binary cell) can be modeled in terms of the classical mass-spring system. Subsequently, the resulting band structure can be studied to have a better understanding of the underlying modes of wave propagation. The detuned dark mode cannot exist independently and must be coupled to the bright mode to get energy from the incident fields. Hence to represent the interference between the bright and dark modes, two masses $\left(m_{1}, m_{2}\right)$ are coupled together through the springs to form an infinitely extended periodic structure, as illustrated in Fig. 5(a). As depicted in Fig. 5(a), the force constant $(\alpha)$ for the all the springs is identical and the period of the structure is $2 a$. Since the equivalent mass-spring oscillator model takes into account the collective response of two vibrating masses $\left(m_{1}, m_{2}\right)$, the displacement of masses can be represented by the following system of coupled differential equations [53]:

$$
\begin{aligned}
& m_{1} \ddot{x}_{i}^{(1)}=\alpha\left(2 x_{i}^{(1)}-x_{i}^{(2)}-x_{i-1}^{(2)}\right), \\
& m_{2} \ddot{x}_{i}^{(2)}=\alpha\left(2 x_{i}^{(2)}-x_{i}^{(1)}-x_{i+1}^{(1)}\right),
\end{aligned}
$$

where $x_{i}^{(1)}$ and $x_{i}^{(2)}$ represent the displacement of the masses $m_{1}$ and $m_{2}$, respectively, inside the $i$ th unit cell of the periodic lattice. Assuming $k$ and $\omega$ to be the wavenumber and the angular frequency, respectively, the time-harmonic oscillations can take the following forms:

$$
x_{i}^{(1)}=A e^{i(k a n-\omega t)}, \quad x_{i}^{(2)}=B e^{i(k a n-\omega t)} .
$$

By replacing the above two solutions in the coupled system of Eq. (3) and by applying the Bloch-Floquet theorem to eliminate the adjacent displacement terms, we can obtained the following two equations:

$$
\begin{aligned}
& m_{1} \omega^{2}=\alpha\left(2 A-B e^{i k a}-B e^{-i k a}\right) / A=2 \alpha(A-B \cos k a) / A, \\
& m_{2} \omega^{2}=\alpha\left(2 B-A e^{-i k a}-A e^{i k a}\right) / B=2 \alpha(B-A \cos k a) / B .
\end{aligned}
$$

The dispersion equation relating the wave numbers to the frequencies can be extracted from Eq. (5) by elimination of the constants $A$ and $B$ after the product of the above two equations has been taken:

$$
\left(1-m_{1} \omega^{2} / 2 \alpha\right)\left(1-m_{2} \omega^{2} / 2 \alpha\right)=\cos ^{2} k a .
$$

Equation (6) can be rearranged in its quadratic form,

$$
\omega^{4}-2 \alpha\left(1 / m_{1}+/ m_{2}\right) \omega^{2}+\left(4 \alpha^{2} / m_{1} m_{2}\right) \sin ^{2} k a=0,
$$

which can be subsequently solved to obtain the band diagram of the spring-mass periodic structure:

$$
\begin{aligned}
\omega^{2}= & \alpha\left[\left(1 / m_{1}+1 / m_{2}\right)\right. \\
& \left. \pm \sqrt{\left(1 / m_{1}+1 / m_{2}\right)^{2}-4 / m_{1} m_{2}\left(\sin ^{2} k a\right)}\right] .
\end{aligned}
$$

Let us first consider the case of equivalent masses $\left(m_{1}=\right.$ $m_{2}$ ), which is analogous to the reflection from the periodic array of identical Lorentz resonators given in Fig. 3. By incorporating the system parameters provided in Table I in the dispersion relation [Eq. (8)], we calculate the band diagram shown in Fig. 5(b). It can be observed that the bands are nearly linear without any band gap between the first two harmonic modes due to the dispersionless nature of propagating waves. This behavior is consistent with the linear reflectance phase depicted in Fig. 3(c).

Consider now the case when the masses in Fig. 5(a) are assumed to be different (or detuned). Solving Eq. (8) yields a dispersion profile that is characterized by splitting of the first two fundamental bands due to the formation of the band gap, as depicted in Fig. 5(b). Within the band gap, only evanescent modes $\left(k_{i} \neq 0\right)$ can exist. This is further explained in the three-dimensional band diagram in Fig. 5(c), which appears to be continuous in the presence 

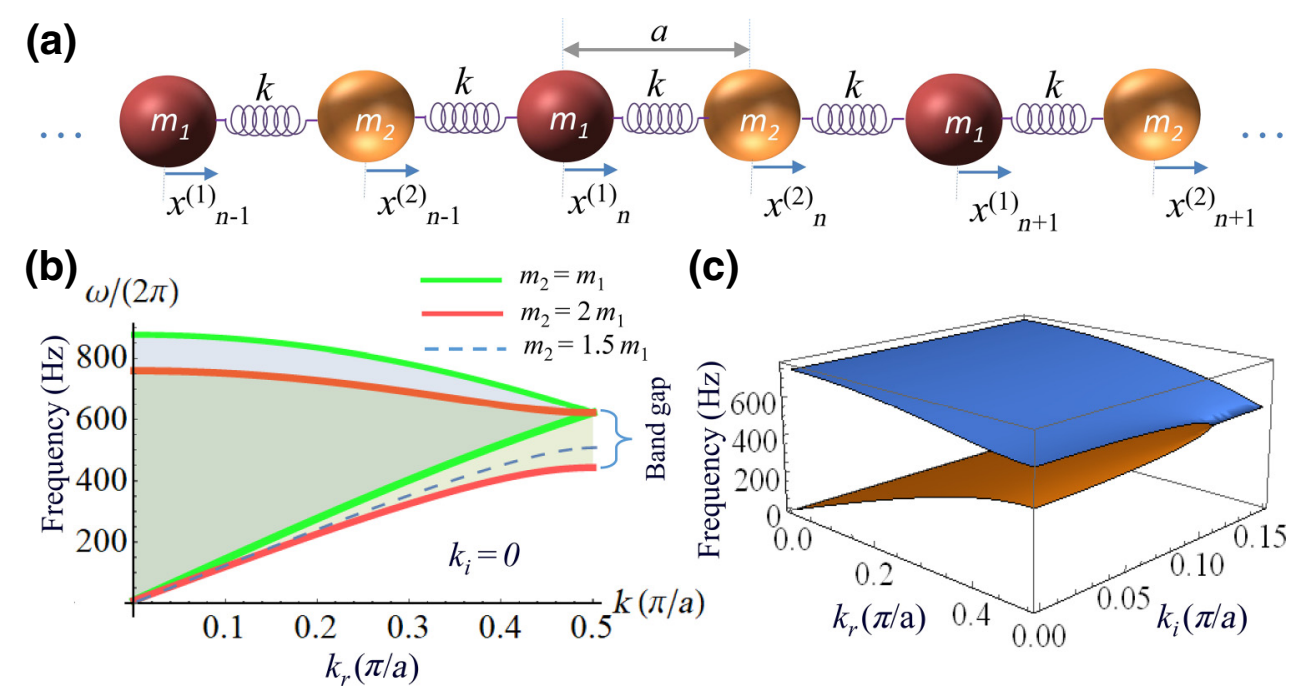

(c)

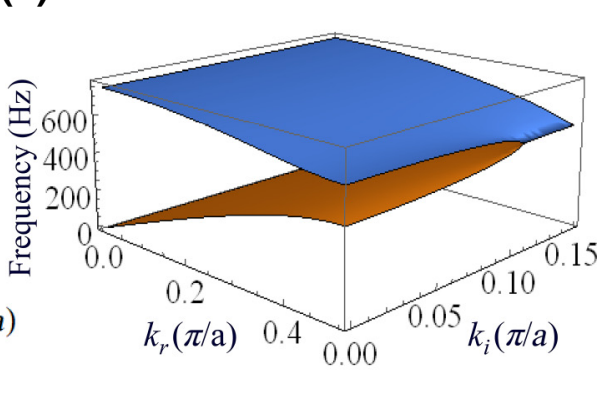

FIG. 5. (a) The classical mass-spring model for understanding the wave propagation in the acoustic metascreen made up of detuned resonator cavities. (b) The Brillouin diagram illustrating the band structure of the mechanical model. (c) The three-dimensional band diagram showing both the real-valued wavenumber $\left(k_{i}\right)$ that corresponds to the propagating waves and the imaginary wavenumber $\left(k_{i}\right)$ representing the evanescent waves.

of the evanescent modes. Note the similarity between the behavior of the mass-spring system and its acoustic binarycell analog (Fig. 4) in the region close to the band gap in which the phase with respect to the change in frequency becomes much steeper $(\delta \phi / \delta f \rightarrow \infty)$. The binary cell as the constituent of the acoustic metasurface is operated in this region of large phase variation. The array of the detuned binary cells that form the metasurface dispersion profile is represented by mass-spring systems with masses having a different ratio (i.e., $m_{2} / m_{1}=2$ ). Furthermore, the dispersion response of the mass-spring system is frequency tunable as shown by the dotted line in Fig. 5(b) for $m_{2} / m_{1}=1.5$.

\section{ACOUSTIC SPATIAL FILTERING THROUGH RESONANT BEAM STEERING}

It is often desired to control the flow of sound of a particular frequency in a desired direction. The conventional way to achieve this is to use a nondispersive inclined planar hard surface (mirror) that reflects incident sound along the surface normal and hence steers it in any particular

TABLE I. The periodically coupled mass-spring parameters for identical masses and detuned masses.

\begin{tabular}{lc}
\hline \hline Parameter & Value \\
\hline$a$ & 1 \\
$m_{1}$ & $1 \mathrm{~kg}$ \\
$m_{2}$ & $2 \mathrm{~kg}$ \\
$\alpha$ & $7.4 \times 10^{6} \mathrm{~N} / \mathrm{m}$ \\
\hline \hline
\end{tabular}

direction. However, this method of acoustic beam steering indiscriminately reflects all the incident waves. Here we introduce the concept of frequency-dependent beam steering that can be used to simultaneously cleanse the unwanted spectral content in the signal and also to direct the acoustic wave in the desired direction. With careful dispersion engineering, such a beam-steering mechanism can be potentially applied to design novel spectral beam splitters and demultiplexers $[54,55]$.

Consider the planar beam-steering screen in Fig. 6(a), which is designed by our arranging phase-distinct binary cells in the $x$ direction. The particular phase dependence that would redirect the reflected wavefronts is first obtained from Eq. (2). The geometrical dimensions of each binary cell needed to achieve the desired phase are obtained from the characteristic curve given in Fig. 4(c). For example, to obtain a $30^{\circ}$ deflection of the reflected wavefront, the scaled-height-to-phase relationship is summarized in Fig. 6(b). The given height distribution results in a linear phase profile along the array axis, as depicted in Fig. 6(c). Here it is emphasized that the beam steering can be achieved over the full horizon due to the fact that the binary cell is capable of achieving a $2 \pi$ reflectance phase change, as shown in Fig. 4(c). Since the binary cell has a finite width $a$, the resulting phase profile is piecewise linear [Fig. 6(c)] and can be designed to be closer to the ideal linear phase (Fig. 2) by our decreasing the binary-cell dimensions. The metasurface is simulated under normally incident planewave condition with use of the full-wave simulator COMSOL MULTIPHYSICS. The simulation results (Fig. 7) show that the scattered field is indeed steered toward a direction of $30^{\circ}$ from the surface normal for the designed 790-mm resonance wavelength. A complete 
(a)

(b)
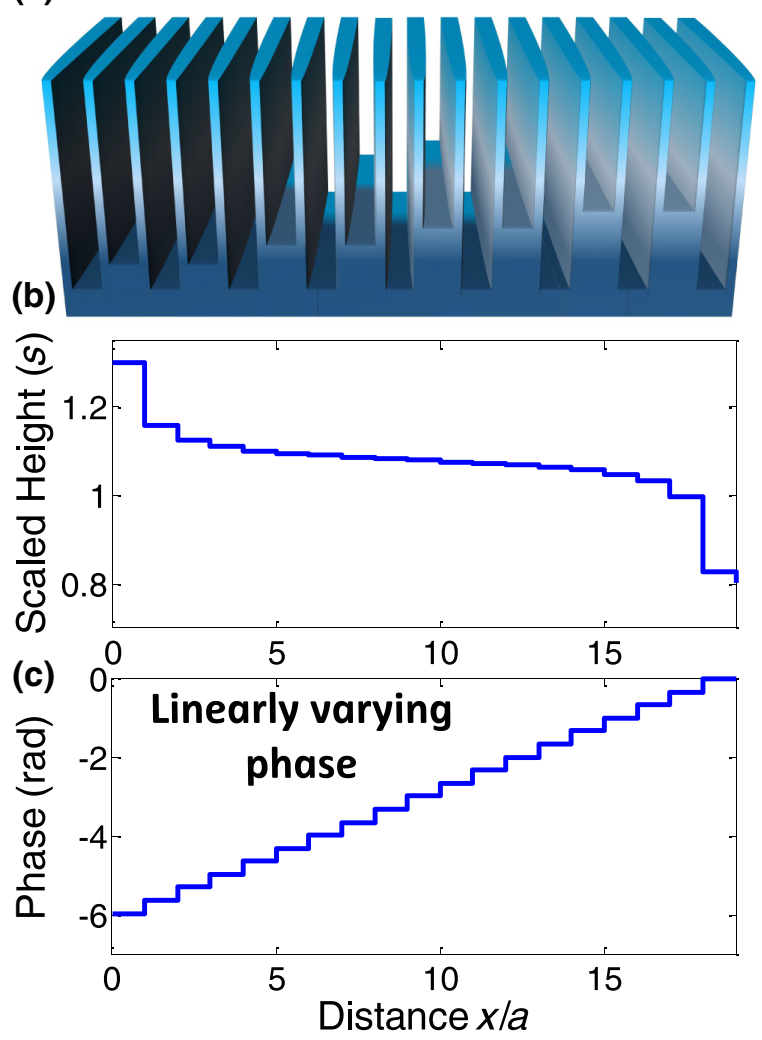

FIG. 6. (a) Planar beam-steering metascreen in which the binary-cell resonators are arranged with different scaled height factors $\left(s_{i}\right)$ along the $x$ direction to provide the necessary phase profile at the aperture. (b) Required scaled height $\left(s_{i=1,2,3, \ldots}\right)$ versus the coordinate $(x / a)$ to achieve beam steering at $30^{\circ}$ from the surface normal and (c) corresponding phase of the reflected sound ranging from 0 to $6 \pi$ (phase unwrapped) versus the coordinate $(x / a)$.

phase wrap allows repetition of the scattered-phase profile and hence offers two additional advantages. Firstly, it allows one to design a large screen without discontinuity.
Secondly, it avoids the unnecessary fringing fields due to phase discontinuity between the adjacent unit cells. To demonstrate the filtering mechanism, the scatteredfield simulations are also performed at $850 \mathrm{~mm}$, which is quite far from the designed frequency. As expected, the metascreen reflects the incident acoustic pressure fields in the incident direction with a planar phase profile [see Fig. 7(b)]. The metascreen bandwidth (i.e., range of frequencies within which the angle of reflection remains constant) is inversely proportional to the sharpness of the associated phase profile. In the resonant structures, the steepness can be reduced by reduction of the quality factor, which one usually does by introducing material damping or using multiple resonances.

\section{VIRTUAL SOUND SCATTERERS FOR ACOUSTIC ILLUSION}

It is possible to construct a Huygens transformational metascreen that would mimic the reflection from an arbitrary shaped object to delude any intruder who sends planar wavefronts for object detection. To form an acoustic illusion, an arbitrary phase profile can be constructed from the dispersion equation (2) and the binary-cell array can be subsequently designed to implement the required phase profile. To demonstrate the illusion acoustics, we first observe the reflected-wavefront signature scattered from a given object and then copy it using the proposed metascreen. Consider the wavefronts reflected from an arbitrary corrugated hard acoustic surface comprising periodic triangles that are tilted at $15^{\circ}$ relative to the horizontal surface, as depicted in Fig. 8(a). For a vertically incident acoustic wavefront, the reflected wavefronts are dispersed away from the surface normal, as depicted in Fig. 8(b). To mimic the reflection from the triangular surface, the reflected phase must compensate for the corresponding phase lead. The corresponding relation between the scaled (a)

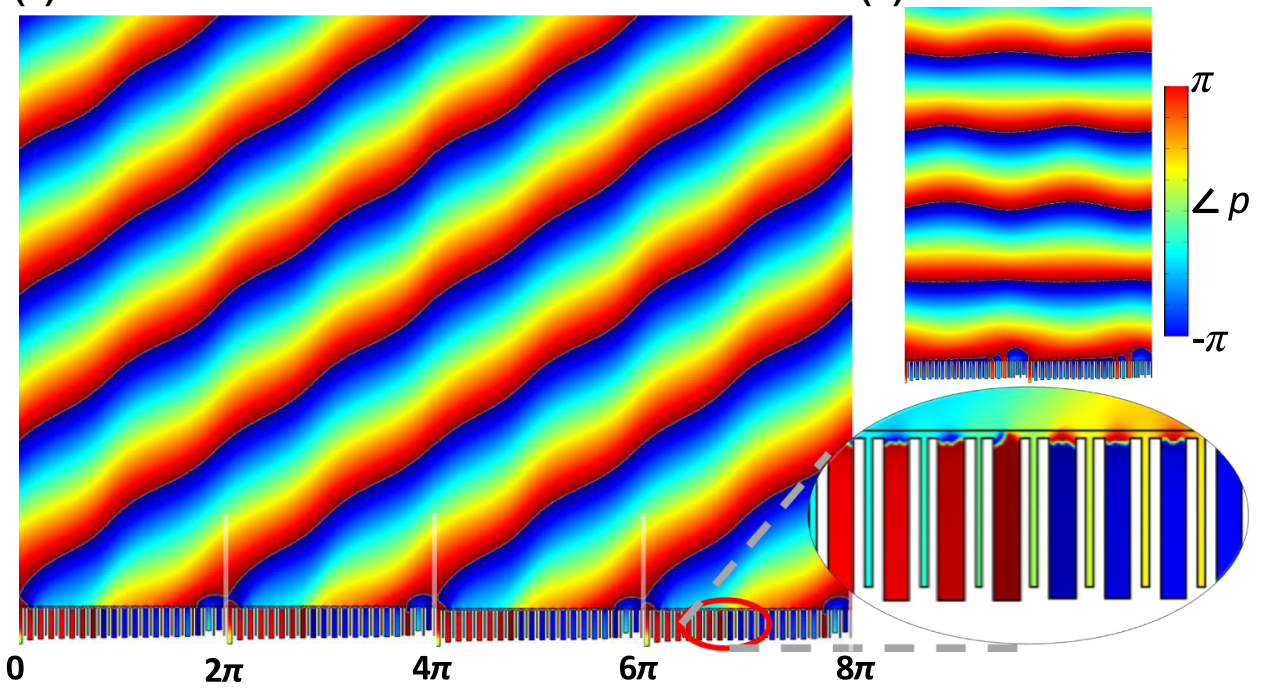

FIG. 7. (a) Reflectance phase of acoustic-scattered-field distribution from the beam-steering metascreen designed for directing the wave at an angle $30^{\circ}$ from the surface normal at an incident wavelength of $790 \mathrm{~mm}$. The designed scaled-height profile in Fig. 6(c) is repeated periodically after a phase-lag interval of $2 \pi$. (b) The scattering characteristics of the designed metascreen in (a) at an incident wavelength of $850 \mathrm{~mm}$ (far from designed wavelength of $790 \mathrm{~mm}$ ). 
(a)

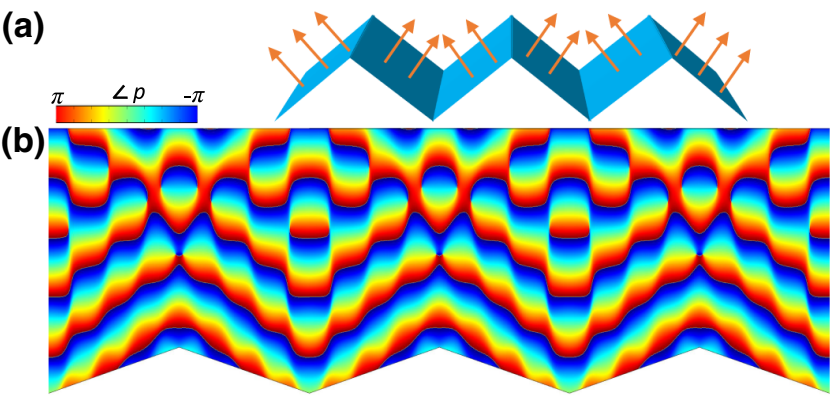

FIG. 8. (a) A periodic array of ridged surface scatterers consisting of triangular bumps. The arrows correspond to the direction of the scattered field. (b) The phase of the reflected scattered pressure field $p$ from the ridge-shaped surface (fields from three periods are shown).

height factors $\left(s_{i}\right)$ and the required phases is summarized in Fig. 9(b). As anticipated, the spatial distribution of the linear phase also forms a triangular profile as depicted in Fig. 9(c). The simulation results (Fig. 10) show that the scattered field at the designed wavelength of $790 \mathrm{~mm}$ indeed resembles the reflections from the actual triangular surface (Fig. 8). Here it is emphasized that the triangular surface is assumed to be periodic to simplify the implementation of boundary conditions in COMSOL MULTIPHYSICS. The method is quite general and can be applied to create finite-sized virtual acoustic scatterers.

\section{RESONANT CARPET CLOAKING}

Carpet cloaks have gained interest in recent years as they offer concealment of objects over a ground plane that would otherwise scatter the incident plane wave [56,57]. The idea is to wrap the object in a transformational screen to virtually restore the planar reflected wavefronts as they are scattered away. Anisotropic cloaking materials often require complex gradient profiles and therefore they are difficult to realize [58]. On the other hand, Huygens surfaces, such as the one designed in the previous section, can be robustly integrated to achieve such carpet cloaking. To implement the cloaking, the dispersive screen is designed so that the phase shifts of the incident wavefronts are compensated. The object we want to conceal here is again the periodic arrangement of triangular bumps, as shown in Fig. 11(a). The triangular surface would reflect the incident wave along two scattering path in the direction that is away from the surface normal. Hence the detected reflections can predict the triangular shape of the surface underneath. Following a procedure similar to the one described in the previous section to design virtual scatterers, a metascreen cloak is designed that can conceal the triangular surface. When the cloak is numerically placed over the triangular surface by implementation of the problem in COMSOL MULTIPHYSICS, near-perfect plane waves are originated from the metascreen and are scattered back in the direction of (a)

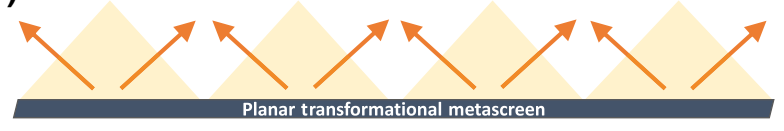

(b)
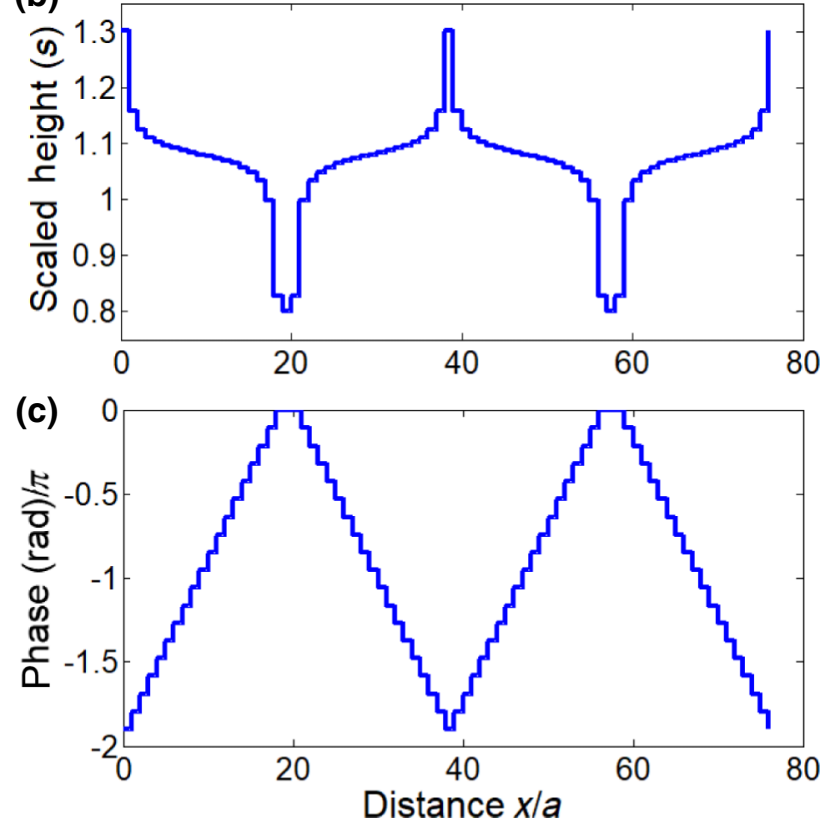

FIG. 9. (a) A planar metascreen to control the scattered field mimicking scattering from triangular bumps. The arrows correspond to the direction of the scattered field. (b) Required scaled height $\left(s_{i=1,2,3, \ldots}\right)$ of resonators and (c) corresponding phase of the reflected field to achieve a scattered field similar to that obtained with periodic triangular bumps. The design is presented for only two periods. The piecewise linear profile is due to the finite size of the binary cell.

the source, as shown in Fig. 11(c). When compared with the scattering from a flat reflector [Fig. 11(c)], the scattered field from the cloak is strikingly similar as it maintains a flat spatial phase profile throughout the far field. The cloaked metascreen can also work for various oblique

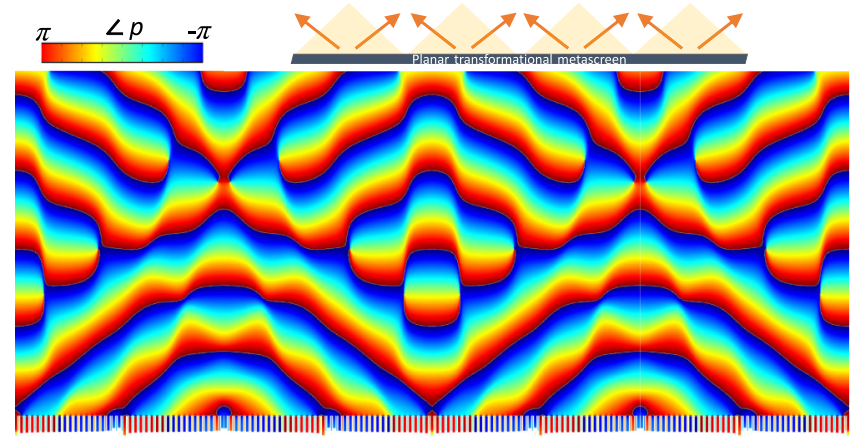

FIG. 10. The phase of the reflected scattered pressure field $p$ from a flat transformational metascreen providing a scattered field equivalent to that obtained with triangular bumps (fields from two periods are shown). 


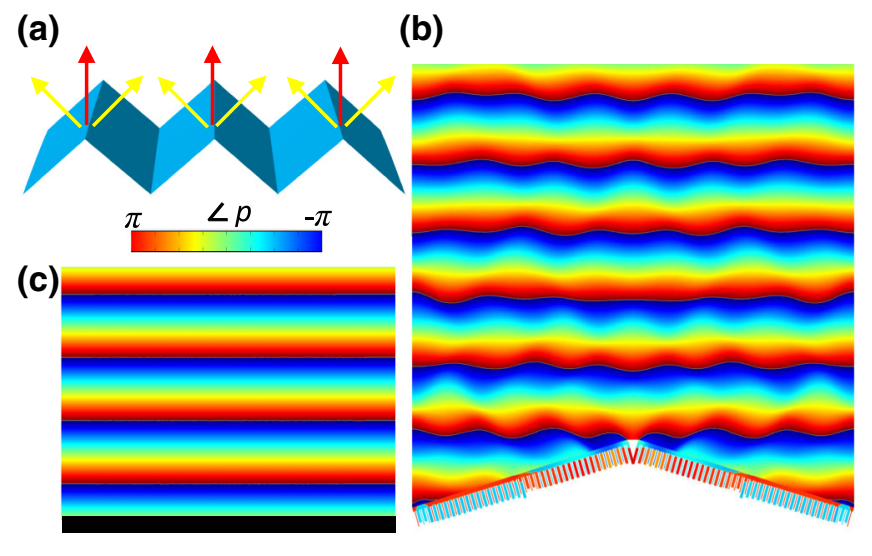

FIG. 11. (a) A periodic array of ridged surface scatterers consisting of triangular bumps. The yellow (red) arrows correspond to the direction of the scattered field without (with) cloaking. (b) The phase of the reflected scattered pressure field $p$ of a ridge-shaped surface with the transformational cloak screen. (c) Reflected scattered field from a flat planar screen.

angles of incidence to delude intruders who come from any direction, as shown for the virtual scatterers in the previous section.

\section{CONCLUSION}

We propose a transformational Huygens metasurface with a planar geometry that can deflect orthogonally incident plane-wave acoustic vectors in any desired direction. To achieve the arbitrary wave reflection, we design the dispersion profile of the metascreen by placing dual strongly coupled Lorentz resonators (termed "binary cells") whose aperture phase can be tuned by virtue of the wave interference with slight geometrical modifications. We derive a generalized dispersion relation for an arbitrary reflected wavefront by invoking the Huygens principle. We also provide a classical mechanical model to represent the acoustic resonators so that the wave propagation in these structures can be better understood. Subsequently, we present full-wave simulation results for a resonant beam-steering metascreen and a carpet-cloaking device. The beam steering is successfully demonstrated at a $15^{\circ}$ tilt with respect to the surface normal. Near-perfect plane-wave scattering along the surface normal is achieved from bump-shaped objects cloaked with an acoustic metascreen. We anticipate use of the dispersion-based metasurface design will open new horizons in the field of deceptive and illusion acoustics.

\section{ACKNOWLEDGMENTS}

W.O. acknowledges the Deanship of Scientific Research, Taibah University, under Grant No. 431/777.
[1] Eli Yablonovitch, Inhibited Spontaneous Emission in SolidState Physics and Electronics, Phys. Rev. Lett. 58, 2059 (1987).

[2] Sajeev John, Strong Localization of Photons in Certain Disordered Dielectric Superlattices, Phys. Rev. Lett. 58, 2486 (1987).

[3] Thomas F. Krauss, M. Richard, and Stuart Brand, Twodimensional photonic-bandgap structures operating at nearinfrared wavelengths, Nature 383, 699 (1996).

[4] Sreela Datta, PhD thesis, School Iowa State University, 1994.

[5] David R. Smith, Willie J. Padilla, D. C. Vier, Syrus C. Nemat-Nasser, and Seldon Schultz, Composite Medium with Simultaneously Negative Permeability and Permittivity, Phys. Rev. Lett. 84, 4184 (2000).

[6] D. R. Smith, J. B. Pendry, and M. C. K. Wiltshire, Metamaterials and negative refractive index, Science 305, 788 (2004).

[7] Paul R. West, Satoshi Ishii, Gururaj V. Naik, Naresh K. Emani, Vladimir M. Shalaev, and Alexandra Boltasseva, Searching for better plasmonic materials, Laser Photon. Rev. 4, 795 (2010).

[8] Zuojia Wang, Feng Cheng, Thomas Winsor, and Yongmin Liu, Optical chiral metamaterials: A review of the fundamentals, fabrication methods and applications, Nanotechnology 27, 412001 (2016).

[9] Vladimir M. Shalaev, Optical negative-index metamaterials, Nat. Photonics 1, 41 (2007).

[10] Nader Engheta, Circuits with light at nanoscales: Optical nanocircuits inspired by metamaterials, Science 317, 1698 (2007).

[11] Boris Gralak, Stefan Enoch, and Gérard Tayeb, Anomalous refractive properties of photonic crystals, JOSA A 17, 1012 (2000).

[12] Masaya Notomi, Negative refraction in photonic crystals, Opt. Quantum Electron. 34, 133 (2002).

[13] Steven A. Cummer, and David Schurig, One path to acoustic cloaking, New J. Phys. 9, 45 (2007).

[14] Richard V. Craster, and Sébastien Guenneau, Acoustic metamaterials: Negative Refraction, Imaging, Lensing and Cloaking (Springer Science \& Business Media, Dordrecht Netherlands, 2012), Vol. 166.

[15] Eric Lheurette, Metamaterials and Wave Control (John Wiley \& Sons, Hoboken, USA, 2013).

[16] Zhengyou Liu, Xixiang Zhang, Yiwei Mao, Y. Y. Zhu, Zhiyu Yang, Che Ting Chan, and Ping Sheng, Locally resonant sonic materials, Science 289, 1734 (2000).

[17] C. G. Poulton, A. B. Movchan, R. C. McPhedran, N. A. Nicorovici, and Y. A. Antipov, in Proceedings of the Royal Society of London A: Mathematical,Physical and Engineering Sciences (The Royal Society, 2000), Vol. 456, p. 2543.

[18] Suxia Yang, John H. Page, Zhengyou Liu, Michael L. Cowan, Che Ting Chan, and Ping Sheng, Focusing of Sound in a 3D Phononic Crystal, Phys. Rev. Lett. 93, 024301 (2004).

[19] Liang Feng, Xiao-Ping Liu, Yan-Bin Chen, Zhi-Peng Huang, Yi-Wei Mao, Yan-Feng Chen, Jian Zi, and 
Yong-Yuan Zhu, Negative refraction of acoustic waves in two-dimensional sonic crystals, Phys. Rev. B 72, 033108 (2005).

[20] Xiangdong Zhang, and Zhengyou Liu, Negative refraction of acoustic waves in two-dimensional phononic crystals, Appl. Phys. Lett. 85, 341 (2004).

[21] Xinhua Hu, Yifeng Shen, Xiaohan Liu, Rongtang Fu, and Jian Zi, Superlensing effect in liquid surface waves, Phys. Rev. E 69, 030201 (2004).

[22] Mohamed Farhat, Sébastien Guenneau, Stefan Enoch, Gérard Tayeb, A. B. Movchan, and N. V. Movchan, Analytical and numerical analysis of lensing effect for linear surface water waves through a square array of nearly touching rigid square cylinders, Phys. Rev. E 77, 046308 (2008).

[23] Jensen Li, and C. T. Chan, Double-negative acoustic metamaterial, Phys. Rev. E 70, 055602 (2004).

[24] Yiqun Ding, Zhengyou Liu, Chunyin Qiu, and Jing Shi, Metamaterial with Simultaneously Negative Bulk Modulus and Mass Density, Phys. Rev. Lett. 99, 093904 (2007).

[25] Sébastien Guenneau, Alexander Movchan, Gunnar Pétursson, and S. Anantha Ramakrishna, Acoustic metamaterials for sound focusing and confinement, New J. Phys. 9, 399 (2007).

[26] Zixian Liang, Tianhua Feng, Fu Liu Shukin Lok, Kung Bo $\mathrm{Ng}$, Chi Hou Chan, Jinjin Wang, Seunghoon Han, Sangyoon Lee, and Jensen Li, Space-coiling metamaterials with double negativity and conical dispersion, Sci. Rep. 3, 1614 (2013).

[27] John Brian Pendry, Negative Refraction Makes a Perfect Lens, Phys. Rev. Lett. 85, 3966 (2000).

[28] Thomas W. Ebbesen, H. J. Lezec, H. F. Ghaemi, Tineke Thio, and P. A. Wolff, Extraordinary optical transmission through sub-wavelength hole arrays, Nature 391, 667 (1998).

[29] Anders Pors, and Sergey I. Bozhevolnyi, Plasmonic metasurfaces for efficient phase control in reflection, Opt. Express 21, 27438 (2013).

[30] Ping-Chun Li, Yang Zhao, Andrea Alu, and Edward T. $\mathrm{Yu}$, Experimental realization and modeling of a subwavelength frequency-selective plasmonic metasurface, Appl. Phys. Lett. 99, 221106 (2011).

[31] Chul-Soon Park, Vivek Raj Shrestha, Wenjing Yue, Song Gao, Sang-Shin Lee, Eun-Soo Kim, and Duk-Yong Choi, Structural color filters enabled by a dielectric metasurface incorporating hydrogenated amorphous silicon nanodisks, Sci. Rep. 7, 2556 (2017).

[32] Abul K. Azad, Wilton J. M. Kort-Kamp, Milan Sykora, Nina R. Weisse-Bernstein, Ting S. Luk, Antoinette J. Taylor, Diego A. R. Dalvit, and Hou-Tong Chen, Metasurface broadband solar absorber, Sci. Rep. 6, 20347 (2016).

[33] Yong Li, and Badreddin M. Assouar, Acoustic metasurfacebased perfect absorber with deep subwavelength thickness, Appl. Phys. Lett. 108, 063502 (2016).

[34] Dan Sievenpiper, Lijun Zhang, Romulo F. J. Broas, Nicholas G. Alexopolous, and Eli Yablonovitch, Highimpedance electromagnetic surfaces with a forbidden frequency band, IEEE Trans. Microwave Theory Techniques 47, 2059 (1999).
[35] Carl Pfeiffer, and Anthony Grbic, Metamaterial Huygens Surfaces: Tailoring Wave Fronts with Reflectionless Sheets, Phys. Rev. Lett. 110, 197401 (2013).

[36] S. A. Schelkunoff, Some equivalence theorems of electromagnetics and their application to radiation problems, Bell System Tech. J. 15, 92 (1936).

[37] Gurkan Gok, and Anthony Grbic, Tailoring the Phase and Power Flow of Electromagnetic Fields, Phys. Rev. Lett. 111, 233904 (2013).

[38] M. Amin, O. Siddiqui, Mohamed Farhat, and A. Khelif, A perfect Fresnel acoustic reflector implemented by a Fano-resonant metascreen, J. Appl. Phys. 123, 144502 (2018).

[39] Marc Dubois, Chengzhi Shi, Yuan Wang, and Xiang Zhang, A thin and conformal metasurface for illusion acoustics of rapidly changing profiles, Appl. Phys. Lett. 110, 151902 (2017).

[40] Boris Luk'yanchuk, Nikolay I. Zheludev, Stefan A. Maier, Naomi J. Halas, Peter Nordlander, Harald Giessen, and Chong Tow Chong, The Fano resonance in plasmonic nanostructures and metamaterials, Nat. Mater. 9, 707 (2010).

[41] Chihhui Wu, Alexander B. Khanikaev, Ronen Adato, Nihal Arju, Ahmet Ali Yanik, Hatice Altug, and Gennady Shvets, Fano-resonant asymmetric metamaterials for ultrasensitive spectroscopy and identification of molecular monolayers, Nat. Mater. 11, 69 (2012).

[42] Fengming Liu, Manzhu Ke, Anqi Zhang, Weijia Wen, Jing Shi, Zhengyou Liu, and Ping Sheng, Acoustic analog of electromagnetically induced transparency in periodic arrays of square rods, Phys. Rev. E 82, 026601 (2010).

[43] Arturo Santillán, and Sergey I. Bozhevolnyi, Acoustic transparency and slow sound using detuned acoustic resonators, Phys. Rev. B 84, 064304 (2011).

[44] M. Amin, A. Elayouch, Mohamed Farhat, M. Addouche, A. Khelif, and H. Bagc, Acoustically induced transparency using Fano resonant periodic arrays, J. Appl. Phys. 118, 164901 (2015).

[45] Na Liu, Lutz Langguth, Thomas Weiss, Jürgen Kästel, Michael Fleischhauer, Tilman Pfau, and Harald Giessen, Plasmonic analogue of electromagnetically induced transparency at the drude damping limit, Nat. Mater. 8, 758 (2009).

[46] Muhammad Amin, Rashid Ramzan, and Omar Siddiqui, Slow wave applications of electromagnetically induced transparency in microstrip resonator, Sci. Rep. 8, 2357 (2018).

[47] Chihhui Wu, Alexander B. Khanikaev, and Gennady Shvets, Broadband Slow Light Metamaterial based on a Double-Continuum Fano Resonance, Phys. Rev. Lett. 106, 107403 (2011).

[48] Sean Victor Hum, and Julien Perruisseau-Carrier, Reconfigurable reflectarrays and array lenses for dynamic antenna beam control: A review, IEEE Trans. Antennas Propag. 62, 183 (2014).

[49] Francesca Venneri, Luigi Boccia, Giovanni Angiulli, Giandomenico Amendola, and Giuseppe Di Massa, Analysis and design of passive and active microstrip reflectarrays, Int. J. RF Microwave Comput.-Aided Eng. 13, 370 (2003). 
[50] Ren Wang, Bing-Zhong Wang, Guo-Feng Gao, Xiao Ding, and Zhi-Peng Wang, Low-profile pattern-reconfigurable vertically polarized end-fire antenna with magnetic-current radiators, IEEE Antennas Wireless Propagation Lett. 17, 829 (2018).

[51] R. de L. Kronig, On the theory of dispersion of x-rays, Josa 12, 547 (1926).

[52] See Supplemental Material at http://link.aps.org/supple mental/10.1103/PhysRevApplied.10.064030 for details about the simulation setup.

[53] Pierre A. Deymier, Acoustic metamaterials and phononic crystals (Springer Science \& Business Media, Dordrecht Netherlands, 2013), Vol. 173.

[54] J. Xu, and J. Tang, Tunable prism based on piezoelectric metamaterial for acoustic beam steering, Appl. Phys. Lett. 110, 181902 (2017).
[55] Katherine F. Woolfe, Jeffrey S. Rogers, Matthew D. Guild, Charles Rohde, Christina J. Naify, and Gregory Orris, Development of the underwater acoustic prism, J. Acoust. Soc. Am. 141, 3699 (2017).

[56] Yihao Yang, Huaping Wang, Faxin Yu, Zhiwei Xu, and Hongsheng Chen, A metasurface carpet cloak for electromagnetic, acoustic and water waves, Sci. Rep. 6, 20219 (2016).

[57] Hui Feng Ma, Wei Xiang Jiang, Xin Mi Yang, Xiao Yang Zhou, and Tie Jun Cui, Compact-sized and broadband carpet cloak and free-space cloak, Opt. Express 17, 19947 (2009).

[58] Wei Xiang Jiang, Jessie Yao Chin, and Tie Jun Cui, Anisotropic metamaterial devices, Mater. Today 12, 26 (2009). 